[3] Z. Frolík, On the topological product of paracompact spaces, Bull. Acad. Polon. Sci. 8 (1960), pp. 747-750.

[4] F. Hausdorff, Mengenlehre, Berlin 1935.

[5] T. Ishii, On product spaces and product mappings, J. Math. Soc. Japan 18 (1966), pp. 166-181.

[6] $\mathrm{Y}$. Katuta, A theorem on paracompactness of product spaces, Proc. Japan Acad. 43 (1967), pp. 615-618.

[7] H. Kok and R. Telgársky, The space of rationals is not absolutely paracompact (this volume).

[8] K. Kuratowski, Topology, Vol. I, New York-London-Warszawa 1966.

[9] A. Lelek, On totally paracompact metric spaces, Proc. Amer. Math. Soc. 19 (1968), pp. 168-170.

[10] - Some cover properties of spaces, Fund. Math. 64 (1969), pp. 209-218.

[11] E. Michael, The product of normal space and a metric space need not be normal, Bull. Amer. Math. Soc. 69 (1963), pp. 375-376.

[12] K. Morita, On closed mappings, Proc. Japan Acad. 32 (1956), pp. 539-543.

[13] - Paracompactness and product spaces, Fund. Math. 50 (1961), pp. 223-236.

[14] - On the product of paracompact spaces, Proc. Japan Acad. 39 (1963), pp. 559-563.

[15] - Products of normal spaces with metric spaces, Math. Annalen 154 (1964), pp. 365-382.

[16] K. Nagami, A note on Hausdorff spaces with the star-finite property II, Proc. Japan Acad. 37 (1961), pp. 189-192.

[17] C. Ryll-Nardzewski, A remark on the cartesian product of two compact spaces, Bull. Acad. Polon. Sci. 6 (1954), pp. 265-266.

[18] Z. Semadeni, Sur les ensembles clairsemés, Rozprawy Mat. 19 (1959).

[19] R. H. Sorgenfrey, On the topological product of paracompact spaces, Bull. Amer. Math. Soc. 53 (1947), pp. 631-632.

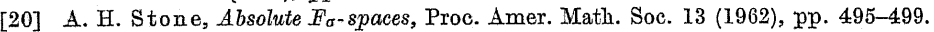

[21] - Kernet constructions and Borel sets, Trans. Amer. Math. Soc. 107 (1963), pp. 58-70; Errata, p. 558.

[22] J. Suzuki, Paracompactness of product spaces, Proc. Japan Acad. 45 (1969), pp. $457-460$.

[23] H. Tamano, On compactifications, J. Math. Kyoto Univ. 1 (1962), pp. 162-193.

[24] R. Telgársky, Total paracompactness and paracompact dispersed spaces, Bull. Acad. Polon. Sci. 16 (1968), pp. 567-572.

[25] - On the product of paracompact spaces, Bull. Acad. Polon. Sci. 17 (1969), pp. 533-536.

[26] M. Tsuda, On the normality of certain product spaces, Proc. Japan Acad. 40 (1964), pp. $465-467$.

INSTITUTE OF MATHEMATICS, POLISH ACADEMY OF SCIENCES, WROCEAW BRANCH.

Reçu par la Rédaction le 28. 4. 1970

\section{The space of rationals is not absolutely paracompact}

by

\section{R. Telgársky (Wrocław) and H. Kok (Amsterdam)}

A Hausdorff space $X$ is said to be paracompact (metacompact) if every open covering of $X$ has a locally finite (point-finite) open refinement.

A Hausdorff space $X$ is said to be totally paracompact (totally meta compact) if every open base of $X$ contains a locally finite (point-finite) covering of $X$.

A family $\Re$ of open sets in $X$ is said to be an outer base of $Y \subset X$ if, for each $y \in Y$ and each open set $G$ in $X$, such that $y \in G$ there exists a $B \in \mathscr{B}$ such that $y \in B \subset G$.

We call a subset $Y$ of $X$ totally paracompact with respect to $X$ if every outer base of $Y$ in $X$ contains a locally finite (with respect to $X$ ) covering of $Y$.

It is easy to prove that if $Y$ is totally paracompact with respect to $X \supset Y$, then $Y$ is a totally paracompact subspace of $X$. A paracompact space $X$ is said to be absolutely paracompact if, for every paracompact space $Y$ such that $X$ is embedded into $Y$ as a closed subspace, $X$ is totally paracompact with respect to $Y$.

For results on totally paracompact spaces we refer to [2] and [5].

In this paper we will prove that a space of E. Michael [3] is not totally metacompact and that the space of the rationals is not absolutely paracompact. It is known that the space of the rationals is totally paracompact, and that the space of the irrationals is paracompact but not totally paracompact (cf. [2]).

Let $\omega^{\omega}$ denote the Baire space of sequences of non-negative integers. It is well known that $\omega^{\omega}$ is homeomorphic to the space of all irrational numbers (cf. [4], p. 143).

Let

$$
D=\left\{f \in \omega^{\omega} \mid \text { 但 } n: \forall k \geqslant n: f(k)=0\right\} \text {. }
$$

Then $D$ is dense in $\omega^{\omega}$ and $D$ is homeomorphic to the space of rationals. 
Let $\Phi$ be the set of all finite sequences of non-negative integers. For $\varphi=\{\varphi(i)\}_{i=0}^{n} \in \Phi$ and $k \in \omega$ we define

$$
\begin{gathered}
h(\varphi)=n+1, \\
B_{\varphi}=\left\{f \in \omega^{\omega} \mid \quad \forall m<l(\varphi): f(m)=\varphi(m)\right\}, \\
D_{\varphi}=D \cap B_{\varphi} \quad \text { and } \quad \overline{\varphi \bar{k}}=\{\psi(i)\}_{i=0}^{n+1} \in \Phi,
\end{gathered}
$$

where $\psi(i)=\varphi(i)$, for $i=0,1, \ldots, n$ and $\psi(n+1)=k$.

For $\varphi$ and $\psi$ in $\Phi$ we define

$$
\varphi<\psi \quad \text { iff } \quad l(\varphi)<l(\psi) \text { and } \nabla k<l(\varphi): \varphi(k)=\psi(k) .
$$

Now let $S$ be the space consisting of the same elements as $\omega^{\omega}$ and having the following (finer) topology: a subset of $S$ is open iff it is of the form $U \cup V$, where $U$ is open in $\omega^{\omega}$ and $\nabla \subset \omega^{\omega} \backslash D$.

It is known that the space $S$ is paracompact (cf. [1], p. 216).

It is easy to see that the relative topology of $D$ is the same both in $\omega^{\omega}$ and in $S$.

The family

$$
\Re_{0}=\left\{B_{\varphi} \mid \varphi \in \Phi\right\},
$$

is an outer base for $D$ in $S$, because it is an open base for the topology in $\omega^{\omega}$.

Now, for every $k>0$ and $\varphi \in \Phi$, we put

$$
B_{\varphi}^{k}=B_{\overline{\varphi 0}} \cup\left(B_{\overline{\varphi k}} \backslash D\right) \quad \text { and } \quad \Re_{B}=\left\{B_{\varphi}^{k} \mid k>0 \text { and } \varphi \in \Phi\right\} .
$$

Clearly, every $B_{\varphi}^{k}$ is an open set in $S$. Moreover, $\mathcal{B}$ is an outer base of $D$ in $S$. For, if $f \in D \cup B_{\varphi}$, then there is a $\psi \in \Phi$ such that $\psi>\varphi, \psi(k)=f(k)$ for every $k<l(\psi)$ and $f(l(\psi))=0$.

Hence $f \in B_{\psi 0} \subset B_{\varphi}^{k}$ for each $k>0$ and $B_{\psi}^{k} \subset B_{\varphi}$.

Now we prove the following

Lemra. If $\mathcal{A} \subset \mathcal{B}$ is a covering of $D$ in $S$, then $\mathfrak{A}$ is not point-finite, i.e. there exists a point $f \in S$ such that $f$ belongs to infinitely many sets from $t$.

Proof. We will define by induction an infinite sequence of sets from $\mathfrak{A}$ having a non-roid intersection.

Let

$$
n_{0}=\inf \left\{l(\varphi) \mid \text { 组 } k: B_{\varphi}^{k} \in \mathcal{A}\right\} .
$$

We choose a $B_{q_{0}}^{k_{0}} \in \mathcal{A}$ such that $l\left(\varphi_{0}\right)=n_{0}$. Then obviously

$$
D_{\overline{q_{0} k_{0}}} \cap \bigcup\left\{B_{\varphi}^{k} \in \mathcal{t} \mid l(\varphi)<l\left(\varphi_{0}\right)\right\}=\varnothing .
$$

If $D_{\overline{\varphi_{0} k_{0}}} \cap B_{\varphi}^{k} \neq \varnothing$ for some $B_{\varphi}^{k} \epsilon \mathcal{A}$ with $l(\varphi)=l\left(\varphi_{0}\right)$, then $D_{\overline{\varphi_{0} k_{0}}} \cap B_{\overline{p 0}} \neq \varnothing$, because $D_{\overline{q_{0} k_{0}}} \cap\left(B_{\overline{q k}} \backslash D\right)=\emptyset$. But then $\overline{\varphi 0}=\overline{\varphi_{0} k_{0}}$ and hence $k_{0}=0$. This is a contradiction. We hare thus shown that

Observe that

$$
D_{\overline{\varphi_{0} k_{0}}} \cap \mathcal{U}\left\{B_{\varphi}^{k} \in \mathcal{H} \mid l(\varphi) \leqslant l\left(\varphi_{0}\right)\right\}=\varnothing .
$$

$$
l(\varphi)>l\left(\varphi_{0}\right) \text { and } B_{\varphi}^{k} \subset B_{\overline{q_{0} k_{0}}} \quad \text { if } \quad B_{\varphi}^{k} \epsilon \in \mathcal{\epsilon} \text { and } B_{\varphi}^{k} \cap D_{\overline{\varphi_{0} k_{0}}} \neq \varnothing \text {. }
$$

Now assume that $n_{t}$ and $B_{\varphi_{t}}^{k_{t}} \in \mathcal{C}$ have been defined in such a way that

$$
l\left(\varphi_{t}\right)=n_{t} \quad \text { and } \quad D_{\overline{q_{t} k_{t}}} \cap \bigcup\left\{B_{\varphi}^{k} \in t \in \mid l(\varphi) \leqslant l\left(\varphi_{t}\right)\right\}=\emptyset .
$$

Now, observe that

$$
l(\varphi)>l\left(\varphi_{t}\right) \text { and } B_{\varphi}^{k} \subset B_{\overline{\varphi_{t} k_{t}}} \quad \text { if } \quad D_{\overline{\varphi_{t} k_{t}}} \cap B_{\varphi}^{k} \neq \emptyset \text { and } B_{\varphi}^{k} \in \mathcal{A} .
$$

Since $D_{\overline{\phi_{t} k_{t}}}$ is covered by $\mathcal{A}$, there is a $B_{\varphi}^{k} \in \mathcal{A}$ such that $D_{\varphi_{t} k_{t}} \cap B_{\varphi}^{k} \neq \varnothing$. Now, let

$$
n_{t+1}=\inf \left\{l(\varphi) \mid \text { 每 } k: B_{\varphi}^{k} \in \mathcal{A} \text { and } D_{\overline{\varphi_{t} k_{t}}} \cap B_{\varphi}^{k} \neq \emptyset\right\},
$$

and choose a $B_{\varphi_{t+1}}^{k_{t+1} \in \mathcal{A}}$ such that $l\left(\varphi_{t+1}\right)=n_{t+1}$ and $B_{\varphi_{t+1}}^{k_{t+1}} \subset B_{\overline{t_{t} k_{t}}}$. It follows from the last inclusion that $\overparen{\varphi_{t+1} k_{t+1}}>\varphi_{t} k_{t}$, and so we have $D_{\overline{\varphi_{t+1} k_{t+1}}} \subset D_{\overline{\varphi_{t} k_{t}}}$.

According to the minimality property of $n_{t+1}$ and the last inclusion, we have

$$
D_{\varphi_{t+1} k_{t+1}} \frown \bigcup\left\{B_{\varphi}^{k} \epsilon \in t \mid l(\varphi)<l\left(\varphi_{t+1}\right)\right\}=\varnothing .
$$

Since we must prove

$$
D_{\varphi_{t+1} k_{t+1}} \frown \bigcup\left\{B_{\varphi}^{k} \in \mathcal{A} \mid l(\varphi) \leqslant l\left(\varphi_{t+1}\right)\right\}=\emptyset,
$$

it is sufficient to show that

$$
D_{\varphi_{t+1} k_{t+1}}^{\widetilde{1}} \frown \bigcup\left\{B_{\varphi}^{k} \epsilon \mathfrak{A} \mid l(\varphi)=l\left(\varphi_{t+1}\right)\right\}=\varnothing .
$$

Suppose, to the contrary, that there exists a $B_{\varphi}^{k} \in \mathcal{A}$ such that

$$
l(\varphi)=l\left(\varphi_{t+1}\right) \quad \text { and } \quad D_{\varphi_{t+1} k_{t+1}}^{\Gamma} \cap B_{\varphi}^{k} \neq \varnothing .
$$

Then $D_{\overline{t_{t+1} k_{t+1}}} \cap B_{\overline{\varphi 0}} \neq \varnothing$, because $D_{\varphi_{t+1} k_{t+1}}^{\tau} \cap\left(B_{\overline{\varphi x}} \backslash D\right)=\varnothing$. But then $\widehat{\varphi^{t}+1} k_{t+1}=\overline{\varphi 0}$, because $l(\varphi)=l\left(\varphi_{t+1}\right)$. Hence $k_{t+1}=0$. This is a contradiction.

From our construction we see that

$$
\varphi_{0}<\varphi_{1}<\varphi_{2}<\cdots
$$

Now let $f \in \mathscr{S}$ be defined by

$$
f(n)=\varphi_{n}(n)
$$


then

$$
f \in \bigcap_{t \in \omega} B_{\varphi_{t}}^{k_{t}} .
$$

So $t$ is not point-finite, and the proof of the Lemma is complete.

As consequences of this Lemma we have the following two theorems:

THEOREM 1. The space $S$ is not totally metacompact.

In fact, $\mathfrak{B}^{\prime}=\mathfrak{B} \cup\{\{x\} \mid x \in \mathbb{S} \backslash D\}$ is an open base of $S$ and $\mathcal{B}^{\prime}$ contains no point-finite covering of $S$, because, by the Lemma, $\mathcal{B}$ contains no point-finite covering of $D$.

THEOREM 2. The space $D$ (which is homeomorphic to the space of the rationals) is not absolutely paracompact.

This is clear, because $D$ is a closed subspace of the paracompact space $S$ and $B$ is a "wrong" outer base of $D$ in $S$.

Remarks. (1) $S$ is a paracompact space with the property that $S^{d}$ (the set of all limit points of $S$ ) is totally paracompact, but $S$ itself is not totally paracompact.

(2) Every $C$-scattered paracompact space is absolutely paracompact [6]. Every paracompact locally compact space and every $F_{\sigma} \cap G_{\delta}$-absolute metrizable space is $C$-scattered paracompact [6]. Hence, there are many absolutely paracompact spaces.

(3) Since there is a homeomorphism $h$ from $\omega^{\omega}$ onto the space of all irrationals in the unit interval $I$ (cf. [4], p. 143), $h(S)$ is a closed subspace of the space $I_{h(D)}$ (cf. [1], p. 216). Since $I_{h(D) \backslash h(S)}$ is a discrete open set, $I_{h(D)}$ is not totally metacompact.

E. Michael (cf. [3] or [1], p. 218) proved that $\omega^{\omega} \times I_{h(D)}$ is not normal. Here, as we have seen above, neither $\omega^{\omega}$ (cf. [2]) nor $I_{h(D)}$ is totally metacompact.

\section{References}

[1] R. Engelking, Outline of General Topology, Amsterdam-Warszawa 1968.

[2] A. Lelek, Some cover properties of spaces, Fund. Math. 64 (1969), pp. 209-218.

[3] E. Michael, The product of a normal space and a metric space need not be normal, Bull. Amer. Math. Soc. 69 (1963), pp. 375-376.

[4] W. Sierpiński, General Topology, Toronto 1961.

[5] R. Telgársky, Total paracompactness and paracompact dispersed spaces, Bull. Acad.

Polon. Sci., Sér. sci. math., astr., et phys. 16 (1968), pp. 567-572.

[6] R. Telgársky, $C$-scattered and paracompact spaces (this volume).

INSTITUTE OF MATHEMATICS, POLISH ACADEMY OF SCIENCES, Wrocław

WISKUNDIG SEMINARIUM DER VRIJE UNIVERSITEIT, Amsterdam

\section{A metrization theorem for developable spaces* \\ by}

James R. Boone (College Station, Tex.)

1. Preliminaries. The usual approach to the problem of metrizability of devrelopable spaces has been through the use of the rich global properties enjoyed by the developable spaces, and the additional assumption of normality. In [2] the application of a significant local property, the first countability of a developable space, was made in giving another (nonnormal) dimension to this problem. The additional global property used in this application was sequential mesocompactness. A family of subsets $\left\{F_{\alpha}: \alpha \in A\right\}$ of a space $X$ is said to be cs-finite if for each convergent sequence, $\left\{P_{i}\right\}$ in $X, F_{a} \cap\left\{P_{i}: i \in N\right\} \neq \emptyset$ for at most finitely many $\alpha \in A$. Accordingly, a Hausdorff space $X$ is called sequentially mesocompact provided: every open covering of $X$ has a cs-finite open refinement.

In this paper, the use of both the local and global properties of developable spaces is made to yield a metrization theorem, Theorem 2.1, for developable spaces which improves both of the following theorems.

TheоReM 1.1 ([1], Theorem 10). A developable space is metrizable if and only if it is collectionwise normal.

THEOREM 1.2 ([2], Theorem 4.2). A developable space is metrizable if and only if it is sequentially mesocompact.

A non-normal simultaneous generalization of sequentially mesocompact spaces and collectionwise normal spaces is introduced.

Definition. A Hausdorff space $X$ is said to have property $(\omega)$ if for each discrete collection of closed sets $\left\{F_{a}: \alpha \in A\right\}$ in $X$, there exists a es-finite collection of open sets $\left\{G_{a}: a \in A\right\}$ such that $F_{a} \subset G_{a}$, for each $\alpha \in A$ and $G_{\alpha} \cap F_{\beta}=\varnothing$, if $\alpha \neq \beta$.

Let $\left\{F_{\alpha}: \alpha \in A\right\}$ be any discrete collection of closed sets in a space $X$. Suppose $X$ is sequentially mesocompact, and consider the open covering $\mathcal{U}=\left\{X-\bigcup_{\gamma \neq \alpha} F_{\gamma}: \alpha \in A\right\}$ of $X$. Let $\subseteq=\left\{G_{\beta}: \beta \in B\right\}$ be a es-finite open

* This work was supported by the Texas Christian University Research Foundation and by Texas A\&M University. 